\title{
Bridging Chemotherapy: Adult Acute Lymphoblastic Leukaemia
}

\author{
Nicolas Boissel and Fabio Ciceri
}

Bridging therapy can be given after leukapheresis and before lymphodepletion during CAR-T cell manufacturing. The primary goal of bridging therapies is to prevent uncontrolled progression of the underlying disease during the manufacturing period before CAR-T cell infusion. Several studies indicate that a high tumour burden is associated with an increased risk of complications after CAR-T cell infusion (Cohen et al. 2019). Therefore, controlling the disease and even possibly decreasing the tumour burden is critical during the manufacturing period. The choice of bridging therapies is essential for the success of the procedure.

Clinical trials of CD19 CAR-T therapy in B-ALL reproducibly report high rates of patient dropout after enrolment due to disease progression or treatment-related complications (Park et al. 2018; Maude et al. 2018). For example, among 75 patients who received a CAR-T infusion in the ELIANA study, 65 (87\%) were treated with bridging chemotherapy between enrolment and infusion, and 10 out of 92 patients enrolled in the trial could not be infused due to significant adverse events or death (Maude et al. 2018). The rate of adult patients infused in the Memorial Sloan Kettering (MSKCC) experience was 65\% (54/83, 65\%) of enrolled patients, mostly due to disease progression and death (Park et al. 2018). This reflects the challenges in clinical management during the 3-6-week period necessary for autologous CAR-T cell preparation (the bridging period).

\footnotetext{
N. Boissel

URP-3518, Institut de Recherche Saint-Louis, Université de Paris, Paris, France
}

Service d'Hématologie Adolescents et Jeunes Adultes, Assistance Publique des Hôpitaux de Paris, Paris, France

e-mail: nicolas.boissel@aphp.fr

F. Ciceri $(\bowtie)$

Vita-Salute San Raffaele University, Milan, Italy

Hematology and Bone Marrow Transplantation Unit, IRCCS San Raffaele Hospital, Milan, Italy

e-mail: ciceri.fabio@hsr.it 
Given that CD19 CAR-T therapies are currently indicated for relapsed/refractory B-ALL patients who have already been exposed to one or more lines of potentially effective therapies, often including combinations of several agents, and that these patients often require therapeutic intervention against rapidly progressive disease or a high tumour burden, the choice of the better approach is not trivial.

Several bridging therapy options now exist, including high-intensity chemotherapy, targeted agents (e.g., TKIs), immunotherapies (e.g., CD-19 or CD22directed), and low-intensity approaches (e.g., vincristine, 6-MP, steroids, thioguanine, etc.). Each approach has pros and cons. For example, high-intensity chemotherapy might be too toxic to allow treatment with CAR-T cells to proceed, while low-intensity approaches might fail in terms of tumour burden reduction.

In addition, treatment with CD19-directed therapies, such as the bispecific T cell engager blinatumomab, might have an impact on the efficacy of subsequent CD19 CAR-T cell therapy (Pillai et al. 2019), and common mechanisms of tumour escape to CD19-directed therapies have now been reported (Boissel 2021). Blinatumomab use was an exclusion criterion from the ELIANA trial (Maude et al. 2018), while it was allowed for patients participating in other similar trials. In the expanded access program for tisagenlecleucel, the overall response rate in patients with prior blinatumomab treatment was $67 \%$ versus $90 \%$ in other patients (Baruchel et al. 2020). However, no univocal data on this important salvage option are available in this setting.

In a recent study, the Memorial Sloan Kettering group reviewed different bridging strategies and outcomes for all patients enrolled in a single-centre, phase 1 trial of CD19-specific CAR-T cells for R/R adult ALL (ClinicalTrials. gov NCT01044069) (Perica et al. 2021). They observed that reductions in disease burden during the bridging period are associated with favourable outcomes after CAR-T therapy and thus suggest that optimal strategies to reduce disease burden during bridging are warranted. They proposed a bridging strategy based on disease burden at the time of the CAR-T therapy decision. They recommended low-intensity therapy for patients with a low tumour burden, low-intensity chemotherapy, or targeted therapy (e.g., inotuzumab) for patients with a high disease burden who are chemorefractory (e.g., partial or short response to prior line of chemotherapy) and unlikely to benefit from highintensity bridging, and a careful evaluation of the risks and benefits of high- vs. low-intensity therapy for patients with high disease burden with expected chemosensitivity (e.g., limited prior chemotherapy exposure, late relapse, or sensitivity to the last line). In fact, not surprisingly, the study showed an increased rate of infections during the bridging period in the high-intensity chemotherapy group.

In conclusion, tumour burden, patient comorbidities, and disease characteristics should tailor the choice of the optimal bridging therapy. The goal of this therapy is not complete disease eradication per se but reduction of tumour burden, preserving 
the patient in good clinical condition for CAR-T cell infusion. The benefits of tumour burden reduction may be twofold, with (1) a reduction in the early risk of adverse events, including cytokine release syndrome and (2) a better outcome after CAR-T cell therapy. Although the role of B-cell-directed therapies should be further and carefully investigated in this setting, mainly to exclude possible interference with CAR-T cell expansion or activity, targeted and low-intensity approaches could be instrumental for this objective. Conversely, high-intensity chemotherapy should be limited to those cases in which the benefit and the probability of achieving a rapid tumour load reduction overcome the risk of infection or another toxic event in the context of a CAR-T-oriented strategy.

\section{Key Point}

- Disease control is necessary before CAR-T cell infusion.

\section{References}

Baruchel A, Krueger J, Balduzzi A, et al. Tisagenlecleucel for pediatric/young adults with relapsed/ refractory acute lymphoblastic leukemia: preliminary report on B2001X study focusing on prior exposure to blinatumomab and inotuzumab. J Clin Oncol. 2020;38(15 Suppl):10518.

Boissel N. ALL in escape room. Blood. 2021;137(4):432-4.

Cohen AD, Garfall AL, Stadtmauer EA, et al. B cell maturation antigen-specific CAR-T cells are clinically active in multiple myeloma. J Clin Invest. 2019;129(6):2210-21.

Maude SL, Laetsch TW, Buechner J, Rives S, Boyer M, Bittencourt H, et al. Tisagenlecleucel in children and young adults with B-cell lymphoblastic leukemia. NEngl J Med. 2018;378:439-48.

Park JH, Rivière I, Gonen M, et al. Long-term follow-up of CD19 CAR-therapy in acute lymphoblastic leukemia. N Engl J Med. 2018;378:449-59.

Perica K, Flynn J, Curran KJ, et al. Impact of bridging chemotherapy on clinical outcome of CD19 CAR-T therapy in adult acute lymphoblastic leukemia. Leukemia. 2021;35(11):3268-71.

Pillai V, Muralidharan K, Meng W, et al. CAR-T cell therapy is effective for CD19-dim B-lymphoblastic leukemia but is impacted by prior blinatumomab therapy. Blood Adv. 2019;3:3539-49.

\section{Further Reading}

Anagnostou T, Riaz IB, Hashmiet SK, et al. Anti-CD19 chimeric antigen receptor T-cell therapy in acute lymphocytic leukaemia: a systematic review and metaanalysis. Lancet Haematol. 2020;7:e816-26.

Brentjens RJ, Rivière I, Park JH, et al. Safety and persistence of adoptively transferred autologous CD19-targeted T cells in patients with relapsed or chemotherapy refractory B-cell leukemias. Blood. 2011;118:4817-28.

Davila ML, Riviere I, Wang X, et al. Efficacy and toxicity management of 19-28z CAR-T cell therapy in B cell acute lymphoblastic leukemia. Sci Transl Med. 2014;6:224ra25. 
Lee DW, Kochenderfer JN, Stetler-Stevenson M, et al. T cells expressing CD19 chimeric antigen receptors for acute lymphoblastic leukaemia in children and young adults: a phase 1 doseescalation trial. Lancet. 2015;385:517-28.

Maude SL, Frey N, Shaw PA, et al. Chimeric antigen receptor T cells for sustained remissions in leukemia. N Engl J Med. 2014;371:1507-17.

Open Access This chapter is licensed under the terms of the Creative Commons Attribution 4.0 International License (http://creativecommons.org/licenses/by/4.0/), which permits use, sharing, adaptation, distribution and reproduction in any medium or format, as long as you give appropriate credit to the original author(s) and the source, provide a link to the Creative Commons license and indicate if changes were made.

The images or other third party material in this chapter are included in the chapter's Creative Commons license, unless indicated otherwise in a credit line to the material. If material is not included in the chapter's Creative Commons license and your intended use is not permitted by statutory regulation or exceeds the permitted use, you will need to obtain permission directly from the copyright holder.

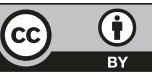

